\title{
The Current Situation and Development of Health Management in China
}

\author{
Li Jiang ${ }^{1^{*}}$, Tao Sha ${ }^{1^{*}}$, Li Ming ${ }^{2}$, Fu Hua ${ }^{1}$, Wang Ke'an ${ }^{2}$, Tian Chuansheng ${ }^{3}$, Wang Longde ${ }^{3}$ \\ 1. School of Public Health, Fudan University, Health Communication Institute of Fudan University, Shanghai 200032, China \\ 2. China Preventive Medicine Association Health Risk Assessment and Control Specialized Committee, Think Tank Research Center for Health \\ Development, Beijing 100176, China \\ 3. China Preventive Medicine Association, Beijing 100710, China
}

\begin{abstract}
Based on a literature search, we selected a total of 17 typical organizations from Jiangsu, Zhejiang, and Shanghai to investigate, including community health service centers, enterprises, health insurance companies, and health management service companies. The results of this study indicated that China has some problems in current health management, for example, community health service centers lack sufficient capacity in terms of personnel allocation and service contents to carry out health management services; foreign-funded enterprises and large state-owned enterprises have carried out health management services with good results, but small and medium-sized enterprises in China lack the independent ability to carry out health management services for their staffs; health insurance companies are reluctant to carry out health insurance services because of deficiencies in profit models, while health management is often provided for high-end customers as an additional option; and health management service companies do not provide real health management services, but only physical examinations. Based on these problems, we put forward a total of eight related policy recommendations and some countermeasures.
\end{abstract}

Keywords: health management; health risk appraisal; life span; basic public health service; clinical preventive service; community health service; health insurance

\section{Introduction}

As China gradually becomes an aging society, the incidence of chronic non-communicable diseases (referred to chronic diseases) is rising. The proportion of chronic diseases in the burden of disease has reached $70 \%$ and the proportion of death causes has increased to $85 \%$ [1]. Facing rising morbidity and mortality rates of chronic diseases and overwhelming medical costs and social burdens, the experience of Western developed countries shows that treating chronic diseases by excessively intensifying treatments and using advanced technologies will result in rapid rises in medical expenses and have little effect on controlling population morbidity. On the contrary, extensive health promotion, prevention services, and health management are relatively low cost and have achieved good results in both economic and social terms [2-4].

Since the concept of health management entered China at the end of the 20th century, health management has made considerable strides in academic disciplines, industrial practices, and personnel training over the past 10 years. According to statistics, China's health management agencies (mainly physical examination institutions) have been growing at a rate of $25 \%$ annually since 2000. A 2013 national survey showed that more than 10000 medical institutions had been set up. In addition to

Received date: November 30, 2016; Revised date: December 20, 2016

Corresponding author: Wang Longde, Chinese Academy of Engineering, Academician; China Preventive Medicine Association, Chariman. Major research fields include epidemiology and public health promotion. E-mail: wangld@nhfpc.gov.cn

${ }^{*}$ Contributed equally author

Funding program: CAE Advisory Project "Strategic Research on National Health and Prevention and Control of Chronic Disease" (2014-XZ-04)

Chinese version: Strategic Study of CAE 2017, 19 (2): 008-015

Cited item: Li Jiang et al. The Current Situation and Development of Health Management in China. Strategic Study of CAE, https://doi.org/10.15302/ J-SSCAE-2017.02.002 
physical examination services, non-medical service organizations that mainly used leisure, beauty, health care, exercise, and rehabilitation as main health management services have also been thriving. Now there are more than 600 thousand non-medical service organizations employing more than 30 million people [5] To reduce expenditure, insurance companies also began paying attention to the health management of insurants by organizing power and resources to provide health management services.

To meet the demand for talent for the development of health management, the Ministry of Labor and Social Security classified "health manager" as a new occupation and released the National Occupational Standards of Health Manager in 2005. The department also utilized social forces to carry out vocational training of health managers and preliminarily explored training modes for them. These measures provided necessary professional technological support for health management work, especially for social forces to carry out health management [6]. Meanwhile, our government listed the health management of different groups as the main basic public health services in the formulation of national basic public health service policies. Therefore, community health service centers have also become an important force in health management. The government has also introduced a series of constructive plans or opinions on policies to promote the launch of prevention and control of chronic diseases and health management. For example, 15 departments such as the Ministry of Health jointly formulated the Work Plan on the Prevention and Control of Chronic Diseases in China (2012-2015) in 2012, which made health management an important part of prevention and control of chronic diseases from the perspective of government documents [7]. To meet the growing demands for health services of people, the State Council issued the Opinions of the State Council on Promoting the Development of Health Services in 2013 and listed health management and health promotion as one of the four core components of the health service industry. Opinions on Accelerating the Development of Commercial Health Insurance and Opinions on Further Strengthening the Patriotic Health Work in the New Period issued in 2014, Plan for the Development of Chinese Medicine Health Services (2015-2020) issued in 2015 by the State Council, and others all emphasized the content of health management and provided policy guarantees for the development of health management in our country.

Although people are paying increasing attention to health management, the current situation of health management's launch, especially in the fields of community health service, enterprise employee health maintenance, and the health insurance industry, still lacks a systematic review. Therefore, the purpose of this study is to scientifically analyze the current status and existing problems of health management in China and put forward policy recommendations so that it can fulfill its role of effectively controlling the burden of chronic diseases and promoting universal health.

\section{Methods}

Through various methods such as a literature search, field research, personal in-depth interviews, panel interviews, and expert argumentation, we conducted an intensive study of primary health care institutions. We conducted 17 field research studies and interviews in Jiangsu, Zhejiang, and Shanghai including community health service centers, enterprises, health insurance companies, and health management service companies based on the sites and target populations of health management. They comprised seven community health service centers (including two each in Jiangsu and Zhejiang, and three in Shanghai), five enterprises (including two medium-sized enterprises and three small-scale companies), two health insurance companies, and three physical examination and health management companies. The survey covered the status quo, service capabilities, and gaps in chronic disease prevention, control, and management as well as available experiences for promotion, existing problems, and feasible solutions.

\section{Results}

Hereinafter, we will introduce the status of health management services collected from the community health service centers, enterprises, physical examination centers, health management companies, and health insurance companies.

\subsection{The situation of health management conducted by community health service centers}

The situation of health management conducted by community health service centers can be summarized in the following aspects.

\subsubsection{Capacities of health management services}

General practitioner teams consisting of general practitioners with different proportions of public health doctors and nurses have been formed. Usually, every four to five general practitioner teams are equipped with one nurse and one public health physician. General practitioner teams constitute the hard core of community health service centers. The work of general practitioners still focuses on traditional modes of treatment with an average of 400 to 500 patients per team per day. This generally reflects that a large amount of medical treatment time is spent in asking questions such as general conditions and medical history, filling out and supplementing the full electronic medical records, and prescribing medicines. The time for doctor's diagnosis is limited. In many cases, even clinical preventive services cannot be completed conscientiously. It follows that the unreasonable composition of the general practitioner team, heavy clinical diagnosis and treatment tasks, and other issues hinder the implementation of health management. 
The larger number of contracted patients per general practitioner team places considerable strain on the management of patients with chronic diseases. A general practitioner usually has about 1000 contracted patients and some even up to 1500 . Relative shortages of staff were common in the seven community health service centers we surveyed. However, there is a big problem in the introduction of talent because the relatively heavy workload and low salary make community health service centers less attractive.

\subsubsection{Content of health management services}

Community residents, especially contracted patients have a very high coverage rate, which is $70 \%$ to $90 \%$ of electronic health records. However, there is a large difference in the update frequency of electronic health records. Updating of electronic health records can only rely on clinical visits besides uniform physical examinations for the elderly. Moreover, none of these community health centers use health records to conduct individual or group-based health risk assessments and lack the ability to conduct health risk assessments.

There are relatively big regional differences in carrying out disease screening. Disease screening is mostly done in the form of a project without a systematic list of diseases, age of beginning, and frequency of screening. These services are mostly based on annual physical examination programs rather than disease features to implement disease screening. Annual physical examination projects are mostly routine items, and some unnecessary checks lead to wastage of medical resources.

Intervention management for risk factors that threaten healthy people is seldom carried out. Health interventions involve use of a single method, which mainly includes health education, regular or irregular health education classes, and others. Some community health service centers carry out tentative work such as sending health tips in text message to contracted patients, giving residents' families pots of oil controlled by government's special projects, and so on. However, none of these health education programs evaluate the effects of these interventions. Many interventions usually rely on special projects, which mostly are short-term and medium-term interventions on a tentative basis or research projects lacking long-term and coherent outcomes. Participating staff are single and fixed so that health education has great limitations.

The management of chronic diseases has been carried out initially. The seven community health service centers task the general practitioner team as a management unit to carry out management of chronic diseases for contracted patients with chronic diseases. The management rate of general community health centers for hypertension can usually be up to $60 \%$. Rates of standardized management for patients willing to accept the management can usually be up to $90 \%$. We found that the large and increasing number of patients with chronic diseases is a serious burden to community health service centers. The doctors' working hours are largely used in outpatient clinics that have high frequencies of monthly visits, especially prescribing drugs rather than drug treatment, grading management, self-management, and other more effective management methods.

Health management and integrated medical and health care models for the elderly are still being tried. The ability of community health service centers to provide daily care, chronic disease management, rehabilitation, health education and counseling, health care, and other services to the elderly need to be improved. We found that a community health service center extends nursing services to residents' families. They also cooperate with nursing homes to provide services such as patient care and regular medical treatment, and try to improve health management for the elderly through an integrated medical and health care model. One of the health service centers also conducts rehabilitation clinics to help patients such as stroke patients after treatment at the top three hospitals. However, day care, full-time care, half-time care, and other forms of care services for the elderly still need to be gradually enriched and improved. Although family-bed services are available at most community health service centers, the limited resources and single category of services cannot meet demand.

Only two of the health service centers surveyed conducted identification services for the constitution of traditional Chinese medicine as part of the content of health records. At present, traditional Chinese medicine services are still mainly based on medical treatment services. Although some communities can carry out various traditional Chinese medicine services including rehabilitation, there is still a long way to go before the concept of "preventive treatment of disease" can be followed. Organizations that carry out identification services for constitution of traditional Chinese medicine have also proposed that community residents have a low response rate to the service. Lack of professionals in the service is another reason why most health service centers do not carry out this work

\subsubsection{Other problems}

Re-education and training principally center on improving clinical skills. Since the service contents provided by community health service centers are mainly diagnosis, treatments, and regular training for doctors, focus on updating to new clinical technologies and training on the theories and methods related to health management is less common. The training contents also emphasize theory and ignore practice so that respondents generally consider that such trainings cannot provide the needed skills. Performance appraisal largely set the number of outpatient as appraisal indicators. The National Basic Public Health Service Regulations have clear evaluation indicators for community health service centers on clinical prevention services and disease management. The document provides reference for all community health service centers. However, these indicators and assessments take the community health service centers as a unit of 
evaluation. Performance evaluation for the doctors within each community health service center is mostly based on clinical performance data such as the number of outpatients. At a doctor's personal level, there is no specific performance appraisal for services such as health management or behavioral interventions except outpatient visits. In the survey, general practitioners' performance awards given by community health service centers are generally evaluated according to medical : public health $=7: 3$, which led to the general practitioner team lacking motivation to implement health management. Meanwhile, inadequate financial input is also a problem faced by various community health service centers.

\subsection{The situation of enterprises to carry out health management}

Because improvement of employees' health status can help improve the productivity of enterprises and reduce medical expenses, enterprises have the will to carry out health management for employees.

\subsubsection{Forms of health management service}

Foreign-invested enterprises and joint ventures adhere to foreign health management concepts and models. In addition to providing employees with traditional medical insurance, medical services and other projects, enterprise management levels also consciously carry out various forms of health maintenance and health promotion projects. However, the health management models of these enterprises are not universally applicable because they require large amounts of financial support from the enterprises themselves. Many middle and small-sized enterprises do not have the ability to provide large and comprehensive health management services for their employees.

\subsubsection{Contents of health management services}

The enterprises investigated are all able to provide basic physical examination services to employees every year. For small enterprises, health-related services to employees are also limited to physical examinations and sport activities. Work related to health management in the two medium-sized enterprises investigated are organizing physical examinations, keeping health records, organizing health lectures, and participating in the organization of health promotion projects. Both enterprises have established employee health records that are based on annual physical examination results. However, due to limitations in their own ability, they do not effectively use these data to implement health interventions. Both enterprises indicated they would irregularly invite relevant experts to hold health lectures with uncertain contents and forms. Health promotion programs include tobacco control and sports activities, such as organizing various ball games, opening sports events, and providing fitness venues and facilities for employees.

\subsubsection{Abilities and questions of health management services}

Small businesses do not have professionals to implement health management. Health maintenance plans for employees are developed and carried out by the human resources department. As two veteran state-owned enterprises with a long history of inheritance, the two medium-sized enterprises surveyed used to have their own corporate hospitals, which were gradually reduced in size afterwards. Now one of them has only one infirmary left, and the other has no infirmary, which was merged into the production safety department.

The enterprises that we researched do not implement systematic and regular health management services. Because small businesses mainly use young workers, they do not show much burden of chronic diseases. The main health threats are mental health problems that are mainly triggered by working pressure. The employees of two medium-sized enterprises were mainly young and middle-aged, and the results of physical examinations showed that the main health threats to employees were high blood fat and high blood pressure. However, in the two mediumsized enterprises, staff that were responsible for follow-up of health management comprised fewer than 10 people. The reason for this has two aspects. On the one hand, the on-the-job staff retire year by year. On the other hand, the existing system cannot provide a promotion channel for practitioners of non-health institution so that graduates of medical college are reluctant to join these enterprises. In terms of funds, enterprises do not have special budgets to purchase health management services for employees. Enterprises can only rely on trade unions to organize relevant activities because of difficulty gaining approval.

\subsection{Physical examination centers, health management companies, and health insurance companies}

Since we entered the 21 st century, the health service industry in our country has been booming. Especially in recent years, the central government has successively issued several guiding documents on promoting the development of the health service industry. Social institutions have also devoted more enthusiasm to the development of the health management industry.

\subsubsection{Contents of health management services in physical} examination centers

In all health service industries, physical examination institutions account for the largest proportion. Physical examination can be understood as the first step in the whole health management service process. Individual health information collected during physical examination is the basis for subsequent health risk assessment and health intervention. Meanwhile, the results of physical examination have a certain authority and opinion guidance. Therefore, physical examination institutions have unique advantages in implementing health risk assessment, health education, and health management for the individual 
according to the results of the physical examination. Persons receiving physical examination are also more willing to accept health education and management on both mental and emotional levels.

The reality is that most physical examination institutions are struggling with daily physical examinations. They provide only result reports to general persons receiving physical examinations. They provide almost no other services and provide at most brief health advice. Meanwhile, our current charge policy does not support physical examination institutions charge fees other than for physical examinations. Therefore, physical examination institutions have neither the ability nor the motivation to conduct more health management projects. Even the physical examination services that have been conducted so far have a series of problems.

(1) The advice provided by physical examinations lacks individual differences. At present, although the domestic physical examination institutions have launched various programs of physical examination packages, classification of packages is mostly based on occupation, spending ability, or family relations rather than the actual health status of users. On the one hand, the majority of healthy people experience unnecessary physical examinations. On the other hand, potential patients lose the opportunity to find diseases early in physical examinations. Some private physical examination institutions employ consulting physicians in the physical examination center. Consulting physicians can give appropriate advice according to the user's appeal. However, they usually suggest that the user add some individual examinations, which is essentially a channel created by physical examination institutions to increase revenue.

(2) The interpretation of the physical examination results is not sufficient. In the reports provided by the physical examination institutions, some comments about important abnormal indexes and suggestions by the medical examiner-in-chief are also included beside the test values and the reference value range of each item. However, these suggestions do not allow users to fully understand their own physical examination results. On the one hand, the medical knowledge of the persons receiving physical examination is limited. On the other hand, the report of the medical examiner-in-chief is usually simple and patterned. The suggestions for the persons receiving physical examination on diet, exercise, and lifestyle are also recapitulative. Regarding group physical examination clients, some physical examination institutions will have a medical examiner-in-chief provide home-based solutions and consultation services for these companies or units.

(3) Management services after the physical examination do not have follow-up. After receiving physical examinations at the physical examination center, the persons' contact is usually interrupted. The physical examination centers also do not find out the populations who have health needs based on the physical examination data to provide some basic health management services.
3.3.2 Contents of health management services in health management companies

Present health management companies are basically evolving from physical examination institutions, or providing services related to physical examinations. Due to the current policy and requirements of consumers, only the physical examination service is recognized by consumers and can receive considerable economic benefits. From the two health management companies we surveyed in Shanghai, the business volume and profits of the physical examination services accounted for more than $80 \%$ of the total business volume and profits. The service targets of health management companies are mainly business groups and high-end business people. In addition to physical examinations, health promotion projects use health consultations, green channels of medical treatments, health lectures, and health promotions as the main contents are their main services. However, people are not willing to pay for health consultations, health lectures, and other services because of people's consumption habits. Therefore, these projects are mainly aimed at attracting consumers and consolidating the customer base. The business of current health management company still follows the approach of check-treat-cure. The core contents of health management services including health risk assessment and risk factor intervention is not involved. The leaders of the health management companies also admit that these services cannot bring economic benefits and customers are not interested in these projects.

\subsubsection{Contents of health management services in insurance companies}

We investigated two large state-owned insurance companies, one of which has a health insurance business and the other plans to implement one. At present, few companies carry out health insurance businesses in China. Currently, China is developing national health insurance dominated by national finance. The development space of commercial medical insurance is very small and many businesses are affiliated to the life insurance department. Besides, health insurance businesses do not bring direct profits to enterprises. The insurance companies set up health insurance mostly to attract customers, increase customer stickiness, and serve other types of insurance. The insurance company provides customers with services related to health management including four aspects. The first is purchasing medical services for customers. The second is keeping the customer's health records. The third is providing green channels of medical treatments for customers who need them such as oral health care, gynecological care, health consultation, and health lectures (including traditional Chinese medicine lectures). The fourth is providing alternative health promotion projects. Some companies now provide health risk assessment services for customers. They purchase health risk assessment software developed by foreign health management companies. Regarding health promotion projects, domestic insurance companies have also introduced 
foreign business philosophies. Insurance companies afford money to provide services for customers. They invite customers to participate in fitness activities, encourage customers to maintain a healthy lifestyle through the Internet of Things, and reward customers with integration. Insurance companies hope to reduce their medical expenses through these health promotion activities. However, generally speaking, there are still many restrictive factors for insurance companies to provide health management for their customers. The work that commercial insurance can do in the field of health management is still limited.

\section{Proposals for development}

Based on the above research results on community health service centers, enterprises, health insurance companies, and health management service agencies, we collated common problems and developed corresponding solutions. We have made the following recommendations for the development of health management.

\subsection{Advocating the concept of life span health management}

Health management should run through a person's entire life rather than from a certain age period, to maximize its effectiveness. In addition to basic public health services (i.e., infants and young children, pregnant women, lying-in women, and the elderly), adolescents, and occupational groups should also be included to provide them with comprehensive health management. For example, the emphasis for adolescents is attending school as their main activity to cultivate good living habits and healthy lifestyles for students, and to improve their health literacy. For occupational groups, we should integrate the three aspects including prevention and control of occupational diseases, workplace health promotion, and health management so that we can move the threshold of prevention of disease to improve the physical and mental health of workers. In addition to clinical prevention services, disease prevention and health promotion services (such as health promotion programs, disease prevention programs, employee assistance programs, etc.) should also be implemented for in-service staff. In short, health management should be implemented as a basic strategy to improve the health literacy of Chinese residents while fully advocating the concept of health management throughout the life span through policy and publicity.

\subsection{Developing appropriate technology for health management and promoting the role of health management in the construction of grading diagnosis and treatment systems}

The government should strengthen the management and support of health management institutions. We need to establish a health management system whose head comprises professional institutions of health promotion and education and whose body comprises community health service centers, schools, enterprises, public institutions, and insurance institutions supplemented by social health management institutions. Based on the actual situation in various regions of our country, especially based on the actual capacity of community health work, the government should encourage health administrative departments at all levels and grassroots service departments to actively develop suitable technologies for health management. To ensure the orderly implementation of health management, the Health Management Work Plan and its supporting documents should be promulgated as soon as possible to clarify the responsibilities of all relevant departments in the work of health management. When conditions are ripe, it is suggested that the National $\mathrm{Pa}$ triotic Health Campaign Committee be renamed the National Health Promotion Committee to bring health management into the health promotion work system to further strengthen the work of nationwide health management. Under the current policy environment of vigorously promoting hierarchical diagnosis and treatment, the promotion role of health management in the construction of hierarchical diagnosis and treatment systems should be taken into consideration. Health management should be integrated into the work of hierarchical diagnosis and treatment at all levels to actively play its role in disease prevention and rehabilitation.

\subsection{Implementing a platform role for community health service centers to establish a community-based grid service mechanism of health management}

Community health service centers directly serve people at a grassroots level. With escalating use of hierarchical diagnosis and treatment, more people will visit community health service centers in the future, compelling the human resources of community health service centers to incline towards diagnosis and treatment in the short term. Therefore, the capabilities of public health services and health management will become more inadequate. We suggest ways to divert the work of health management from community health service centers. Services that require a high degree of professionalism including vaccination, maternal and child health, and others can be reserved in community health service centers, while work that can be implemented by health managers can be delivered to third-party companies. In addition, strengthening the power of first diagnosis in community health service centers provides convenience for health management. The community health service center will master and manage the health information of residents in the area as the terminal for health services. Both health management companies and health insurance companies need to use the community health service center as a transit platform to provide services to residents. The advantage is that the data model for sharing can be standardized 
and greater data security ensured. Community health service centers will be responsible for evaluation and appraisal of contractors after outsourcing health management services and other services (Fig. 1).

\subsection{Carrying forward the advantages of traditional Chinese} medicine and integrating "preventive treatment of disease" into health management

As the unique classic medical theory in our country, the concept of "preventive treatment of disease" is similar to the concept of modern health management that originated in the West. Therefore, we can start from giving a leading role to "preventive treatment of disease" in modern health management and then combine traditional Chinese medicine with modern Western medicine to promote the development of health management. People's health status can be assessed through identification services for the constitution of traditional Chinese medicine. Individual health intervention can be carried out through health care and nourishing the life of traditional Chinese medicine. We can cultivate and improve the health concepts and health literacy of the masses by popularizing traditional Chinese medicine, and ultimately achieve the purpose of enhancing physical fitness, preventing diseases, and increasing people's well-being.

\subsection{Establishing mechanisms of health information sharing and promoting standardization of health management service processes}

The primary problem of health management is the collection and utilization of health information, which is also the basis for follow-up work. The current task is to speed up the exploration and establishment of a responsible department for the integration and management of health information as well as formulation of work procedures and related systems. The government should promote the sharing of health information both inside and outside institutions and prevent the leakage and commercialized abuse of personal information. The government can try to upload and store health information from each unit in a qualified information management organization. Downstream Health Management can download and use this health information with the consent of the owner of the information. The administration is responsible for supervising the security and ethics issues of information storage.

In addition, health risk assessment is an important link, which has high technical requirements in health management. Scientific and accurate assessment is the only way to carry out health management as well as the key to improving the efficiency of health management. However, present health risk assessment tools on the market are a mess and lacking effective evaluation and supervision. Therefore, the technical norms of health risk assessment need to be formulated as soon as possible. Based on evidence-based medicine, we should encourage the development of health risk assessment tools that are suitable for the Chinese population. We also recommend that a scientific and effective health risk assessment should be actively conducted in the community.

We should strictly regulate the content of health management, processes, and major technical means when vigorously developing the health management industry. We also need to clarify the access standards, service targets, service items, business models, and requirements of employees and others in various institutions so as to strengthen the supervision of health management services.

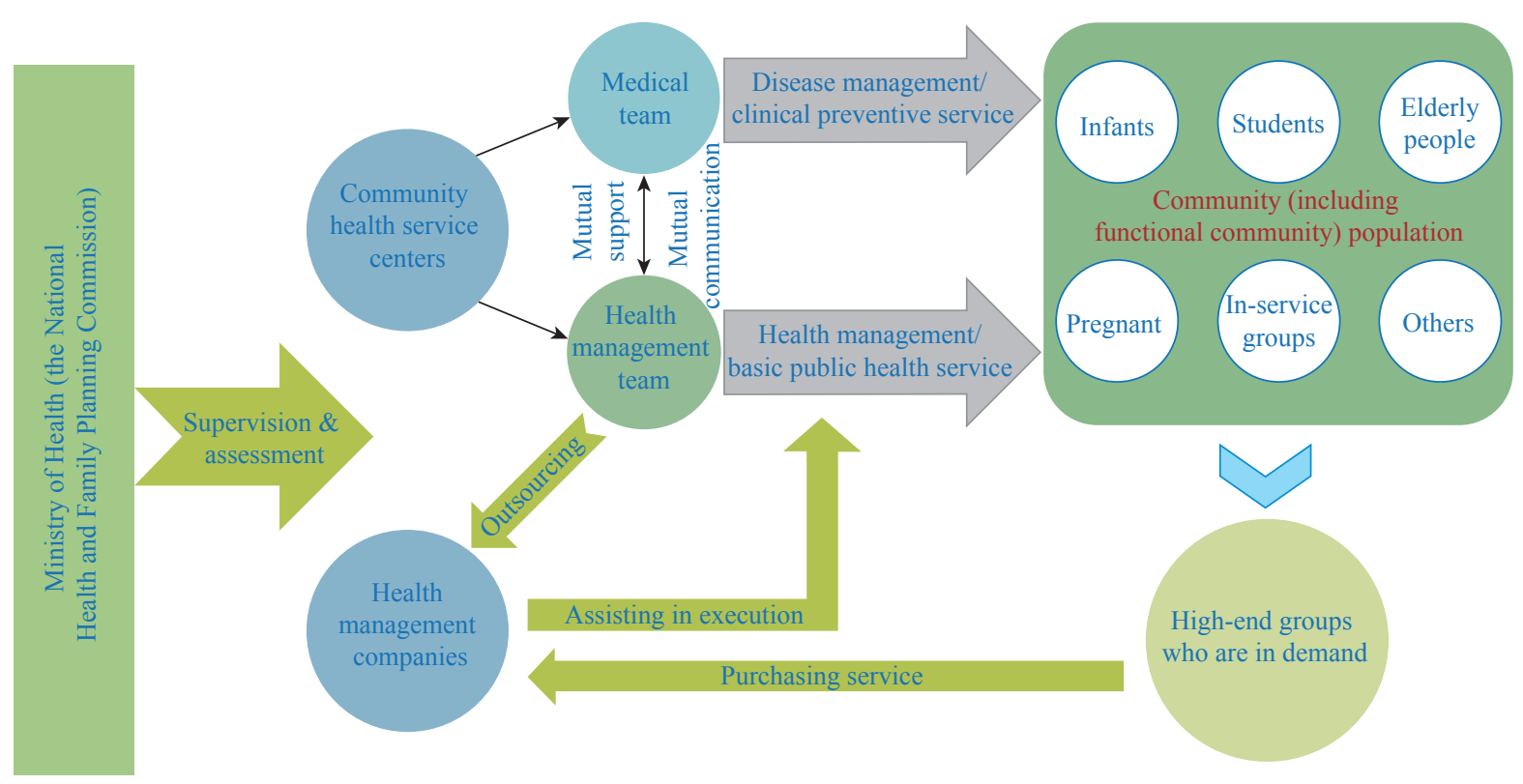

Fig. 1. Basic service architecture of health management. 
4.6 Increasing the training of health management personnel and enhancing the capacity of personnel to establish a health management team with Chinese characteristics

There is a problem of institutions underperforming and staffing shortages at all levels of health management. To solve the problem, we should start with two aspects. Firstly, we should increase the training of existing employees in the improvement of occupational skills and post abilities. We should make clear the responsibilities and scope of work of staff including health manager, nutritionist, psychological counselor, and others in health management services to improve their operational and service skills. These staff cannot start their career unless obtaining practicing qualifications. We should encourage practitioners in the health and medical system, especially older nursing staff to accept training and to transfer to health management professionals. Secondly, we should increase the training of professionals. The government should encourage medical colleges and universities to set up health management courses or even majors and vigorously train graduates of junior college and regular college. Meanwhile, the government should encourage and guide other medical graduates to engage in work related to health management. Competent authorities need to start from the actual situation in our country, take the actual demand of the masses as the principle and the market as the guideline, and make clear the competency requirements of practitioners of health management to establish a personnel access system so that those who enter the industry can truly exert their professional expertise.

\subsection{Straightening out healthy management of toll collection channels to solve the problem of inadequate funding for health management}

At present, there are no special funds for health management. The funds from state finance mainly include funds for basic public health services and project funds from various state ministries and local governments. The part from social funds mainly includes income from physical examinations. However, little income from physical examinations is actually devoted to the field of health management. Insufficient investment severely limits the sustainable development of health management. To ensure the long-term health management work and funding stability, it is recommended to introduce health insurance into grassroots health management services and set community health service centers as a platform to explore a mode in which health insurance pays the cost of health management to reduce medical expenditure. Meanwhile, the government should actively introduce social resources and open up channels for funding of health management. On the one hand, health management should be included as a means of proactive prevention in the risk control measures of medical insurance. The introduction of a market model should be accelerated. Government procurement through third-party services should be sparkplugged; these ways are aimed at complementing the deficiency of health management services from community health service centers and enterprises, and public institutions. Health management plays a role of reducing expenditure for health insurance, so theoretically medical expenses managers and payers have incentives and willingness to pay for health management. Including health management into the health care budget is both conducive to developing an overall plan for national health care expenditures and to improving the functional setting (expenditure + savings) of the health insurance system. It is recommended to set up special funds, that is, national health insurance, to cover the cost of health management services. The funds of state health insurance come from the expenditure of the state's basic public health services and national health insurance. On the other hand, the government should actively promote a combination of commercial insurance and health management, provide policy support for health insurance business, and encourage commercial insurance to participate in the health care services system. Both national health insurance and commercial health insurance should be based on the community health service centers as the operating platform to serve the community residents (Fig. 2).

\subsection{Improving the construction of health promotion and} health management systems in the workplace and improving the health of the working population

In terms of policies and systems, we should actively promote the construction of health service systems in the workplace. Health management in developed countries is also derived from health promotion in the workplace. In the decades before China's reform and opening up, most of its health needs were solved in the workplace. Prevention services also ran through the support and service systems of enterprise workers. However, in recent years, the staff's health-related services have been unattended after the cancellation of the health service system in the work-

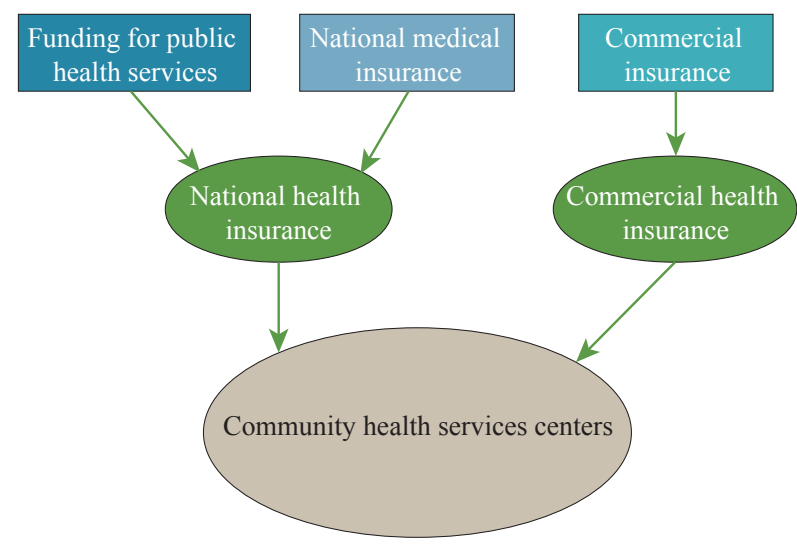

Fig. 2. Combination of commercial insurance and health management. 
place. With rapid changes in lifestyle, the epidemic level of risk factors for chronic diseases, as well as the morbidity and prevalence rates of chronic diseases in the working population are rising rapidly. In addition, when workers retire, they also bring the "health burden" they have accumulated over the years into society, which is equal to a large number of unhealthy people being transported into the social insurance system. Therefore, it is suggested that enterprises should be encouraged to rebuild or increase their input of the health service system in the workplace. The government should provide policy support, assistance in terms of capacity, and use health promotion and health management as a lever to mobilize the health dividend for entire social groups.

\section{References}

[1] National Health and Family Planning Commission of the PRC China health and family planning statistical yearbook 2015 [M]. Beijing: Peking Union Medical College Press, 2015. Chinese.

[2] Koffman M D M, Lanza A, Campbell K P. A purchaser's guide to clinical preventive services: A tool to improve health care coverage for prevention [J]. Preventing Chronic Disease, 2008, 5 (2): 1-9. Chinese.

[3] Schill A L, Chosewood L C. The NIOSH total worker health program: An overview [J]. Journal of Occupational and Environmental Medicine, 2013, 55 (12 suppl.): 8-11. Chinese.

[4] Martinez-González N A, Berchtold P, Ullman K, et al. Integrated care programmes for adults with chronic conditions: A meta-review [J]. International Journal for Quality in Health Care, 2014, 26(5): 561-570.

[5] Bai S Z, Wu L X, Chen G, et al. Innovative theory and practice of health management in China $[\mathrm{J}]$. Chinese Journal of Health Management, 2014, 8 (2): 75-78. Chinese.

[6] Wang P Y. Current situation, problems and prospect of health management theory and practice $[\mathrm{J}]$. Chinese Journal of Health Management, 2015, 9 (1): 2-6. Chinese.

[7] Ministry of Health of the PRC, National Development and Reform Commission of the PRC, Ministry of Education of the PRC, etc. The notice of Ministry of Health of the PRC etc. on the issuing the plan for the prevention and treatment of chronic non-communicable diseases in China (2012-2015) [J]. Gazette of the Ministry of Health of the PRC, 2012 (5): 31-36. Chinese. 